\title{
RAB11A mediates the proliferation and motility of esophageal cancer cells via WNT signaling pathway
}

\author{
Danyi Zhao', Bing Wang1ه and Huawei Chen² \\ 'Department of Gastrointestinal oncology, The Second Hospital of Dalian Medical University, Dalian City, Liaoning Province, 116023 , China; ${ }^{2}$ Cell \\ Biology Laboratory, Dalian Medical University, Dalian City, Liaoning Province, 116044, China
}

Esophageal cancer (EC) recently has become a common malignancy of digestive system worldwide. RAB11A is a critical member of the small GTPases superfamily and was reported to affect a variety of cellular functions. However, its potential effects on EC progression and the specific regulatory mechanisms are still unclear. In this study, RAB11A was upregulated in human EC tissues and cells and predicted poor diagnosis. RAB11A expression was correlated with clinical-pathological features including pTNM stage $\left(P=0.001^{*}\right)$ and recurrence $\left(P=0.000^{* *}\right)$ in patients with EC. Furthermore, RAB11A knockdown decreased the proliferation, migration, and invasion of EC cells via WNT pathway in vitro. Subsequently, the in vivo experiments confirmed that RAB11A contributed to EC tumor growth via WNT pathway. Therefore, these results provided evidence showing that RAB11A could promote the progression of EC via WNT pathway and might serve as a promising therapeutic target for EC treatment.

Key words: esophageal cancer, RAB11A, proliferation, migration, WNT pathway

Received: 15 June, 2020; revised: 04 September, 2020; accepted: 30 October, 2020; available on-line: 16 December, 2020

$\triangle$ e-mail: BingWangfgj@163.com

Abbreviations: EC, esophageal cancer; ESCC, esophageal squamous cell carcinoma; OSR, overall survival rate; MTT, 3-(4,5-dimethyl-2-thiazolyl)-2,5-diphenyl-2-H-tetrazolium bromide NSCLCs, non-small cell lung cancer cells; RIPA, radioimmunoprecipitation; TC, thyroid cancer; PFA, paraformaldehyde; TBST, Tris-buffered saline with Tween

\section{INTRODUCTION}

Esophageal cancer (EC) is a common malignancy of digestive system worldwide, ranked seventh in incidence and sixth in mortality (Lutfi et al., 2020). There are over 200000 new cases of EC in China every year, and the 5 -year overall survival rate (OSR) of EC patients is still less than 25\% (2002). Esophageal squamous cell carcino$\mathrm{ma}$ (ESCC) is the main histological type of EC, accounting for nearly $90 \%$ of cases in China (Nam et al., 2020). Although there are many treatment methods for EC, the prognosis of patients is poor due to high metastasis ability and local lymph node metastasis (Su et al., 2020). It is well-accepted that targeted therapy is a promising prospect for EC treatment (Wu et al., 2020). Therefore, to improve the survival rate of patients with EC, novel and effective drugs, and particularly, therapeutic targets are urgently needed.

RAB11A is a critical member of small GTPases superfamily and is involved in a variety of cellular functions, such as the regulation of membrane vesicular sorting and transport, ciliogenesis, and autophagy (Jiang et al., 2017).
It was also reported that RAB11A controls intracellular transport of innate immune receptor TLR4 (Husebye et al., 2010). Knockdown of RAB11A can cause serious human diseases, such as the defective formation of basolateral microvilli in neonatal intestinal diseases, infertility, tumors, etc. (Knowles et al., 2015; Feng et al., 2017; Dobiliene et al., 2018). RAB11A is highly expressed in various tumor tissues, such as thyroid cancer, breast cancer, and lung cancer (Calhoun et al., 1998; Palmieri et al., 2006; Dong et al., 2017; Jiang et al., 2017).

For decades, the possible regulatory mechanism of RAB11A on tumor progression has been revealed (Palmieri et al., 2006). RAB11A could affect the motility of non-small cell lung cancer cells (NSCLCs) by regulating YAP (Dong et al., 2017). RAB11A was also found to be dysregulated in EC, but its clinical significance remains unclear (Robert et al., 2005; Shrestha et al., 2020). Additionally, the specific regulatory mechanism is also unclear. A previous study showed that RAB11A was regulated by miR-150 and affected the proliferation of thyroid cancer (TC) cells via WNT pathway (Bai et al., 2017). Based on these conclusions, a possibility that RAB11A may contribute to EC progression through WNT pathway was raised.

In this study, we reported the abnormally high RAB11A expression in human EC tissues and cell lines. RAB11A expression was also related to the clinical-pathological features of EC patients. RAB11A knockdown suppressed the proliferation, migration and invasion of EC cells via WNT pathway in vitro, and inhibited EC tumor growth in vivo. Therefore, our findings confirmed the regulatory role of RAB11A in EC progression and revealed the mechanism.

\section{MATERIALS AND METHODS}

Antibodies, primers and shRNA plasmids. The following antibodies were used: anti-RAB11A (for IHC assays, 1:100 dilution, for immunoblot assays, 1:1000 dilution, ab128913, Abcam, Cambridge, UK), anti-Ki67 (for IHC assays, 1:500 dilution, for immunoblot assays, 1:2000 dilution, ab16667, Abcam), anti-Cyclin D1 (1:2000 dilution, ab16663, Abcam), anti-MMP2 (1:1000 dilution, ab97779, Abcam), anti-MMP9 (1:1000 dilution, ab38898, Abcam), anti-GSK3 $\beta$ (1:1000 dilution, ab32391, Abcam), anti-p-GSK3 $\beta$ (1:500 dilution, ab131097, Abcam), anti- $\beta$-catenin (1:1000 dilution, ab16051, Abcam), and anti- $\beta$-actin (1:2000 dilution, ab8226, Abcam).

The quantitative real-time PCR assay was performed with RAB11A primers (forward, 5'-TCAAAGGCTGGGTGGATA-3' and reverse, 5'-GCACCACCCAACCAGAAG-3'). GAPDH was amplified as an 
internal control (forward, 5'-GAGTCAACGGATT'TGGTCGT-3' and reverse 5'-T'TGATT'T'TGGAGGGATCTCG-3'). RAB11A shRNA (pLKO.1-puro-Rab11a) and control plasmids were purchased from Addgene.

Human samples. The 50 pairs of human EC tissues and para-carcinoma tissues were acquired from patients diagnosed in The Second Hospital of Dalian Medical University. Patients' consent was obtained before the experiment. All procedures were performed in accordance with the standards upheld by the Ethics Committee of The Second Hospital of Dalian Medical University (Approval no. 2017014) and the 1964 Helsinki Declaration and its later amendments for ethical research. The clinical-pathological characteristics, such as patient age, gender, p'NM stage, lymph node metastasis, and recurrence were listed in Table 1.

Immunohistochemical (IHC) assays were performed to detect the expression of RAB11A in EC tissues. Briefly, paraffin-embedded tissues were cut into $4 \mu \mathrm{m}$ sections. Then, the slices were fixed in $4 \%$ paraformaldehyde (PFA) for $1 \mathrm{~h}$ at room temperature and subsequently immersed in 2\% BSA for 30 minutes at room temperature. The slices were incubated with anti-RAB11A antibodies at room temperature for 2 hours. Subsequently, the sections were stained with biotinylated secondary antibody for another 1 hour and visualized with diaminobenzidine. RAB11A was mainly localized in the cytoplasm of EC tissues. The staining scores for RAB11A were evaluated as follows: 0 for negatively stained cells; 1 for $1-20 \%$ stained cells; 2 for $21-60 \%$ stained cells; 3 for $61-100 \%$ stained cells. The intensity of staining was evaluated as 0 (no staining), 1 (weak staining), 2 (medium staining) and 3 (strong staining). The expression levels of RAB11A were scored and calculated using the following formula: score $=$ the score of staining intensity $\times$ the score of stained cells percentage. The samples were identified as "high-expression" with score $\geq 3$, while the others were considered "relatively low-expression".

Cell culture and transfection. The human EC cell lines, TE-10 and Eca109, obtained from ATCC were maintained in Dulbecco's Modified Essential Medium (DMEM), with 10\% fetal bovine serum (FBS, Gibco, CA, USA), in a humidified incubator with $5 \% \mathrm{CO}_{2}$.

For RAB11A knockdown, Lipofectamine 2000 (11668019, Invitrogen, CA, USA) was used to transfect RAB11A shRNA plasmids into TE-10 and Eca109 cells. For the control group, the shRNA targeting sequence was nonsense and could not target any intracellular RNAs. TE-10 cells with shRAB11A stable transfection were screened with puromycin and used for the further in vivo experiments.

Quantitative PCR assays. Extraction of total RNA from tissues or cells was conducted with Trizol (15596026, Invitrogen, CA, USA). Then, RNA was reverse-transcribed using M-MLV reverse transcriptase (M1701, Promega, Wisconsin, USA). SYBR Ex Taq kit (638319, Takara, Japan) was used for quantitative PCR and GAPDH was used as the control.

Immunoblot assays. Proteins from EC cells or tissues were extracted with Radioimmunoprecipitation (RIPA) Buffer (9800, Cell Signaling, Danvers, MA). Then, SDS-PAGE was performed and the proteins were transferred onto polyvinylidene fluoride (PVDF) membranes, which were incubated in $5 \%$ milk in Tris-Buffered Saline with Tween (TBST) buffer and then probed with indicated primary antibodies at room temperature for 2 hours. Then, the PVDF membranes were incubated with HRP-conjugated secondary antibodies for 45 minutes and analyzed using an ECL system.

Table 1. Relationship of RAB11A and clinicopathological characteristics in 50 patients with esophageal caner

\begin{tabular}{|c|c|c|c|c|}
\hline Characteristics & Number of patients & $\begin{array}{l}\text { RAB11A } \\
\text { Low expression ( } \leq \text { medin) }\end{array}$ & $\begin{array}{l}\text { RAB11A } \\
\text { High expression (> medin) }\end{array}$ & $P$ value \\
\hline Number & 50 & 25 & 25 & \\
\hline Ages (years) & & & & 0.258 \\
\hline$<55$ & 26 & 11 & 15 & \\
\hline$\geq 55$ & 24 & 14 & 10 & \\
\hline Gender & & & & 0.239 \\
\hline Male & 18 & 7 & 11 & \\
\hline Female & 32 & 18 & 14 & \\
\hline pTNM stage & & & & $0.001^{*}$ \\
\hline $\mathrm{T} 2$ & 28 & 20 & 8 & \\
\hline $\mathrm{T} 3 / \mathrm{T} 4$ & 22 & 5 & 17 & \\
\hline Tumor grade & & & & 0.225 \\
\hline Low & 16 & 6 & 10 & \\
\hline High & 34 & 19 & 15 & \\
\hline Lymph node metastasis & & & & 0.390 \\
\hline Yes & 21 & 12 & 9 & \\
\hline No & 29 & 13 & 16 & \\
\hline Recurrence & & & & $0.000^{* *}$ \\
\hline Yes & 29 & 7 & 22 & \\
\hline No & 11 & 18 & 3 & \\
\hline
\end{tabular}

${ }^{*} P<0.05,{ }^{* *} P<0.001$ 
Colony formation assays. EC cells were seeded into 6 -well culture plates at a density of $1000 /$ per well. $5 \mu \mathrm{l}$ transfection reagent, $1 \mu \mathrm{g}$ plasmid and $250 \mu \mathrm{L}$ of serumfree medium were mixed for 5 minutes at room temperature. 5 minutes later, the mixed solution was added to the 6-well culture plate and incubated for 6 hours. Then, the cells were cultured for 14 days (medium was replaced every 2 days). The cells were fixed with PFA for 30 minutes at room temperature, rinsed in PBS and stained with $0.2 \%$ crystal violet at room temperature for 30 minutes. The colonies were quantified manually.

MTT assays. EC cells were seeded into 96-well plates at a density of approximately 1000 cells each well and incubated for 24 hours. 3-(4,5-dimethyl-2-thiazolyl)-2,5diphenyl-2-H-tetrazolium bromide (MT'T) was added and incubated for 4 hours at room temperature and the culture medium was removed. Subsequently, the cells were washed with PBS and dimethyl sulfoxide (DMSO) was added. The absorbance was analyzed with a microplate reader at $490 \mathrm{~nm}$ wavelength.

Wound-healing assays. Cell migration was assessed using wound-healing assays. In brief, cells transfected with the indicated plasmids were scraped with a $20 \mu \mathrm{L}$ tip to make a wound, followed by rinsing. Subsequently, the cells were maintained in the complete culture medium to stimulate wound healing. Photographs were captured at 0 hours and 24 hours time-point to evaluate the migration of EC cells.

Cell invasion assays. Cell invasion was measured using Transwell chambers ( $8 \mu \mathrm{m}$ pore size; Corning) with Matrigel. After transfection, cells in FBS-free DMEM medium were seeded into the upper chamber with $10 \%$ Matrigel. The complete medium was added to the bottom chamber. After incubation for 24 hours, the cells in the upper chamber were stimulated to invade into the bottom chamber. Then, the upper chamber medium was removed, and the lower chamber cells were fixed in PFA and visualized with $0.2 \%$ crystal violet. The invasive cells were counted under a microscope.

$A$

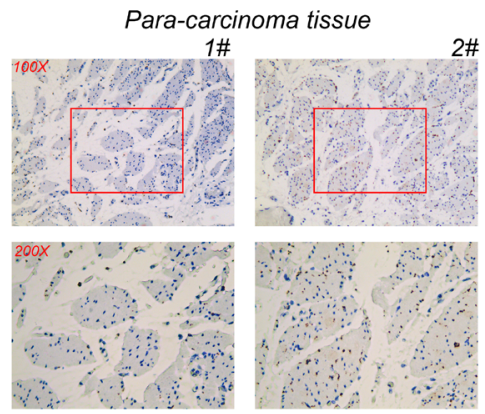

Tumor growth assays. The animal experiments were approved by the Laboratory Animal Ethics Committee of Dalian Medical University. 6 female Balb/C nude mice aged 6 weeks were used in each group, and all mice were given adequate food and water and did not die normally. The mice were sacrificed by cervical dislocation with heartbeat check before the tumor tissue was removed. Adequate humanitarian measures were taken. For RAB11A depletion, TE-10 cells were stably infected with control or RAB11A shRNA lentivirus. Subsequently, approximately $5 \times 10^{5} \mathrm{TE}-10$ cells infected with the indicated lentivirus were implanted into athymic nude mice subcutaneously. Tumor volume was assessed every week. After 6 weeks, the tumors were extracted and photographed, and the tumor growth curves were calculated.

Statistics. Statistical analysis was performed with GraphPad 6.0 software. All results were shown as mean \pm S.D. The correlation between clinical features of EC patients and RAB11A expression levels was analyzed using the $\chi^{2}$ test. Student's $t$-test was used for statistical comparisons. *indicated $P<0.05$, **indicated $P<0.01$, and $* * *$ indicated $P<0.001$, which was also considered as a statistically significant difference.

\section{RESULTS}

\section{RAB11A expression was up-regulated in human esophageal cancer (EC) tissues and predicted poor prognosis}

The RAB11A expression was assessed in EC and para-carcinoma tissues from 50 patients. IHC staining results showed that the expression of RAB11A in paracarcinoma tissues (Fig. 1A, left) was lower than that in EC tissues (Fig. 1A, right). In addition, quantitative PCR assays were performed to detect the mRNA levels of RAB11A in EC and para-carcinoma tissues and showed the higher mRNA levels of RAB11A in EC tissues than
$B$

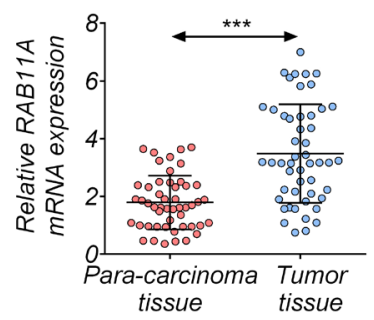

C

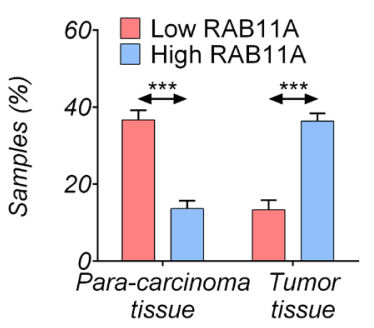

Tumor tissue

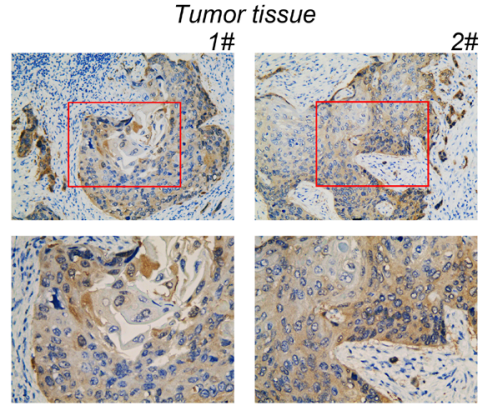

$D$

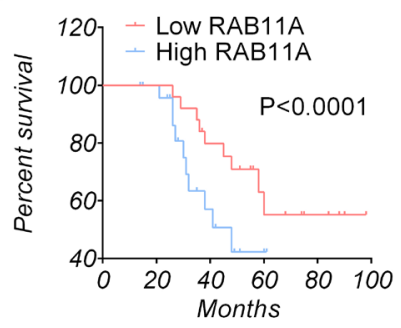

Figure 1. RAB11A was upregulated in human EC tissues and predicted poor prognosis.

(A) Immunohistochemical assays showed RAB11A expression in EC and para-carcinoma tissues, and representative figures were showed (100x and 200x magnification). (B) Quantitative PCR assays were performed to detect the mRNA levels of RAB11A in EC and para-carcinoma tissues. (C) The distribution of patients in the high or low RAB11A expression group. (D) Kaplan-Meier survival analysis was performed to assess the overall survival rate. Results were presented as mean \pm S.D., ${ }^{* * *}$ indicates $P<0.001$. 
A
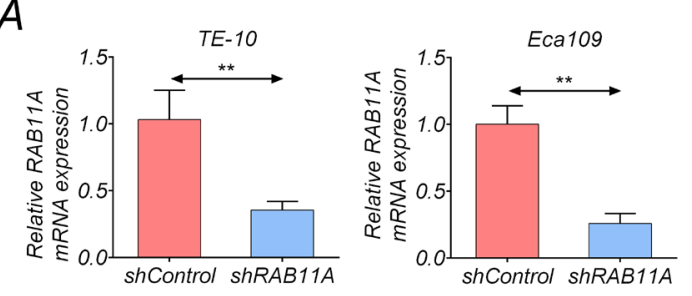

$B$

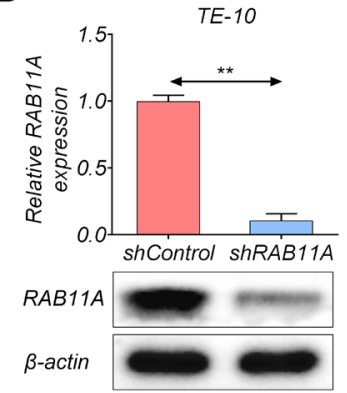

$E$
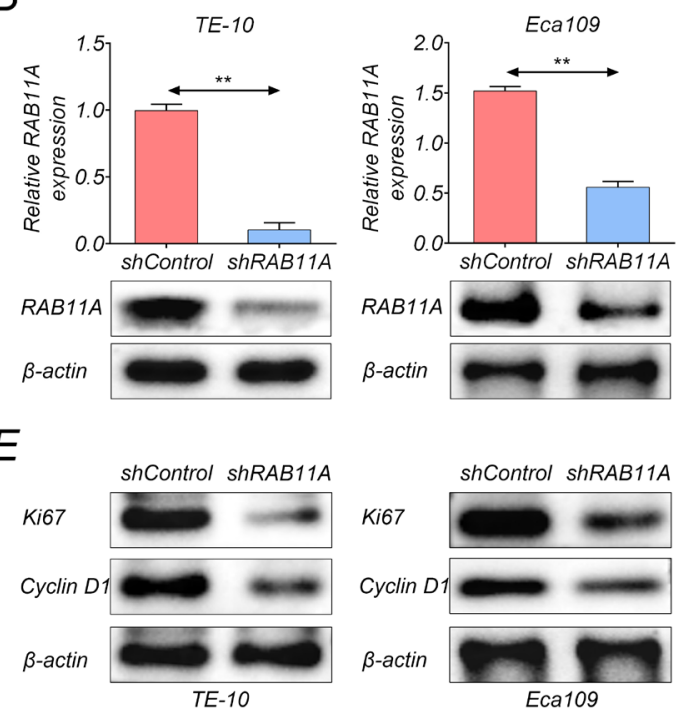

C
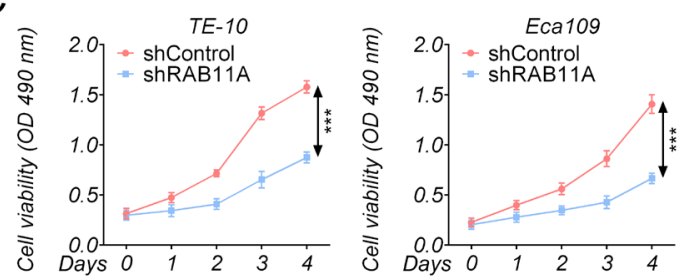

D
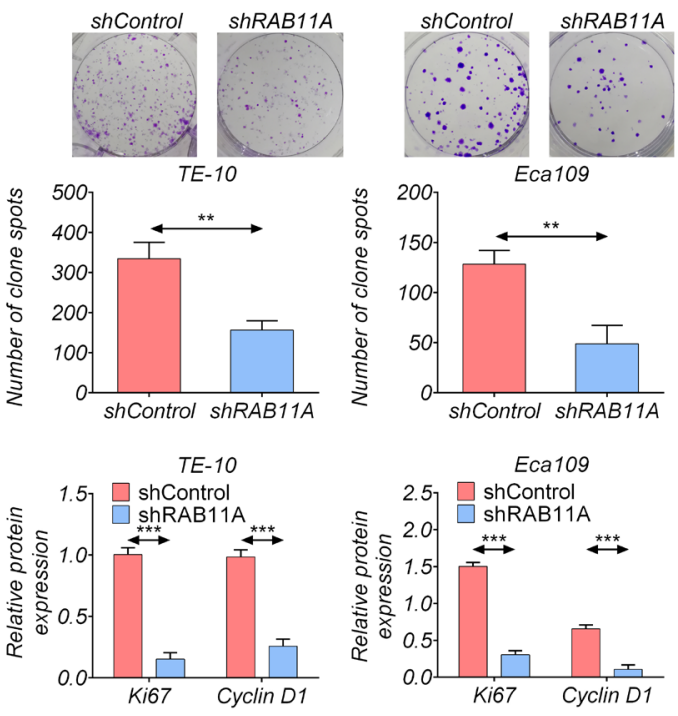

Figure 2. RAB11A depletion suppressed the proliferation of EC cells in vitro.

(A) Quantitative PCR assays were performed to detect the mRNA levels of RAB11A in TE-10 or Eca109 cells. (B) Immunoblot assays were conducted to detect the expression of RAB11A in TE-10 or Eca109 cells. (C) MTT assays were performed to evaluate the proliferation of TE-10 or Eca109 cells. (D) Colony formation assays were conducted to evaluate the colonies number in TE-10 or Eca109 cells. (E) The expression of Ki67 and Cyclin D1 in TE-10 or Eca109 cells was detected using immunoblot assays. All experiments were repeated three times, and results were presented as mean \pm S.D., **indicates $P<0.01,{ }^{* * *}$ indicates $P<0.001$.

para-carcinoma tissues (Fig. 1B). Based on the RAB11A mRNA levels, 50 patients were divided into 2 groups, low RAB11A group ( $\leq$ median) and high RAB11A $(>$ median) group ( $\mathrm{n}=25$ in each group). Figure $1 \mathrm{C}$ shows that the number of patients with high RAB11A expression was larger than that with low RAB11Aexpression. The K-M survival analysis showed that the expression of RAB11A closely correlated with the survival rate of patients with EC and that high RAB11A expression predicted poor survival of the patients (Fig. 1D).

\section{The expression of RAB11A was significantly related to the clinical-pathological features of EC patients}

The correlation between RAB11A expression and clinical-pathological features including age, gender, pTNM stage, tumor grade, lymph node metastasis, and recurrence, was analyzed. There was no significant correlation between RAB11A expression and age $(P=0.258)$, gen$\operatorname{der}(P=0.239)$, tumor grade $(P=0.225)$, nor lymph node metastasis $(P=0.390)$. However, RAB11A expression was related to pTNM stage $\left(P=0.001^{*}\right)$ and recurrence $\left(P=0.000^{*}\right)$ of EC patients (Table 1$)$. To further confirm the correlation between RAB11A expression and clinical features, we performed multivariate Cox regression analysis. We also noticed that RAB11A expression correlated with the pTNM stage $\left(P=0.043^{*}\right)$ and recurrence $\left(P=0.048^{*}\right)$ of patients with EC, consistent with the previous data (Table 2). Therefore, RAB11A expression was related to the clinical features of EC patients.

\section{RAB11A depletion decreased the proliferation of EC cells in vitro}

To further clarify the involvement of RAB11A in EC progression, shRNA plasmid targeting RAB11A was used to lower its expression in human EC cell lines, TE-10 and Eca109. The transfection efficiency was confirmed by quantitative PCR assays (Fig. 2A) and western blotting (Fig. 2B).

Subsequently, MT'T and colony formation assays were performed to explore the effects of RAB11A on the proliferation of TE-10 and Eca109 cells in vitro. MT'T assays showed that the OD value at $490 \mathrm{~nm}$ was obviously decreased in TE-10 and Eca109 cells after RAB11A was knocked down compared to the shControl group (Fig. 2C). Similarly, RAB11A knockdown led to the reduction in colony number of TE-10 and Eca109 cells (Fig. 2D). The expression levels of Cyclin D1 and Ki67, two cell cycle- and proliferation-related markers, were detected in TE-10 and Eca109 cells after transfection with RAB11A shRNA or control plasmids. RAB11A depletion decreased the expression of Cyclin D1 and Ki67 in TE-10 and Eca109 cells as compared to the shControl group (Fig. 2E).

\section{RAB11A depletion decreased the migration and invasion of EC cells}

Subsequently, the involvement of RAB11A in the migration and invasion of EC cells was determined. RAB11A depletion resulted in the decreased migratory 
Table 2. The multivariate Cox regression analysis of patients with esophageal cancer (EC).

\begin{tabular}{|c|c|c|c|c|c|c|c|c|}
\hline & \multirow{2}{*}{ B } & \multirow{2}{*}{ SE } & \multirow{2}{*}{ Wald } & \multirow{2}{*}{$\mathrm{df}$} & \multirow{2}{*}{ Sig. } & \multirow{2}{*}{$\operatorname{Exp}(B)$} & \multicolumn{2}{|c|}{$95.0 \% \mathrm{Cl}$ for $\operatorname{Exp}(\mathrm{B})$} \\
\hline & & & & & & & Bottom & Upside \\
\hline RAB11A exoression & 0.630 & 0.309 & 4.145 & 1 & $0.042^{*}$ & 1.877 & 1.024 & 3.440 \\
\hline Ages & -0.449 & 0.551 & 0.664 & 1 & 0.415 & 0.638 & 0.217 & 1.879 \\
\hline Gender & 0.482 & 0.536 & 0.807 & 1 & 0.369 & 1.619 & 0.566 & 4.634 \\
\hline pTNM stage & -1.333 & 0.658 & 4.108 & 1 & $0.043^{*}$ & 0.264 & 0.073 & 0.957 \\
\hline Tumor grade & -0.949 & 0.694 & 1.868 & 1 & 0.172 & 0.387 & 0.099 & 1.510 \\
\hline Lymph node metastasis & 0.487 & 0.534 & 0.832 & 1 & 0.362 & 1.628 & 0.571 & 4.638 \\
\hline Recurrence & 1.945 & 0.985 & 3.902 & 1 & $0.048^{*}$ & 6.995 & 1.015 & 48.195 \\
\hline
\end{tabular}

${ }^{*} P<0.05$

abilities of TE-10 and Eca109 cells as compared to the shControl group (Fig. 3A). Similarly, Transwell assays showed that RAB11A knockdown decreased the invasive abilities of TE-10 and Eca109 cells (Fig. 3B). We then assessed the expression of MMP2 and MMP9, two invasion-related markers, in TE-10 and Eca109 cells after transfection with RAB11A shRNA or control plasmids. The results revealed that RAB11A depletion led to the decreased expression of MMP2 and MMP9 in TE-10 and Eca109 cells (Fig. 3C). Therefore, these data showed that RAB11A promoted the migration and invasion of EC cells.

\section{RAB11A mediated WNT pathway in EC cells}

The following analysis evaluated the regulatory mechanisms underlying the involvement of RAB11A in EC progression. By performing immunoblot assays, the protein levels of GSK3 $\beta$, phosphorylated GSK3 $\beta$ (pGSK3 $\beta$ ), and $\beta$-catenin in TE-10 and Eca109 cells were assessed after RAB11A depletion. Interestingly, our data showed that RAB11A depletion decreased the level of phosphorylated GSK3 $\beta$, and downregulated $\beta$-catenin in TE-10 and Eca109 cells, respectively (Fig. 4). Taken together, RAB11A could activate GSK3 $\beta-\beta$-catenin axis in EC cells.

\section{RAB11A promoted EC tumor growth via WNT pathway in mice}

Finally, we checked whether RAB11A stimulated EC tumor growth using an animal model. Briefly, TE-10 cells with vector or RAB11A shRNA lentivirus were subcutaneously implanted into athymic nude mice. The expression level of RAB11A was obviously decreased in RAB11A depletion group as compared to shControl group as shown by immunoblot assays (Fig. 5A). The representative images of tumors were shown in Fig. 5B, with smaller tumor size in shRAB11A group as compared to shControl group. Though there were no obvious differences in mice weight between shControl and shRAB11A groups (Fig. 5C), the tumor volume in shRAB11A group was significantly decreased compared to shControl group (Fig. 5D). IHC assays showed that RAB11A, Ki67, and $\beta$-catenin expression levels were decreased in shRAB11A group as compared to shControl group (Fig. 5E).

\section{DISCUSSION}

EC has become a common malignancy of the digestive tract that affects about 300000 people worldwide
$A$
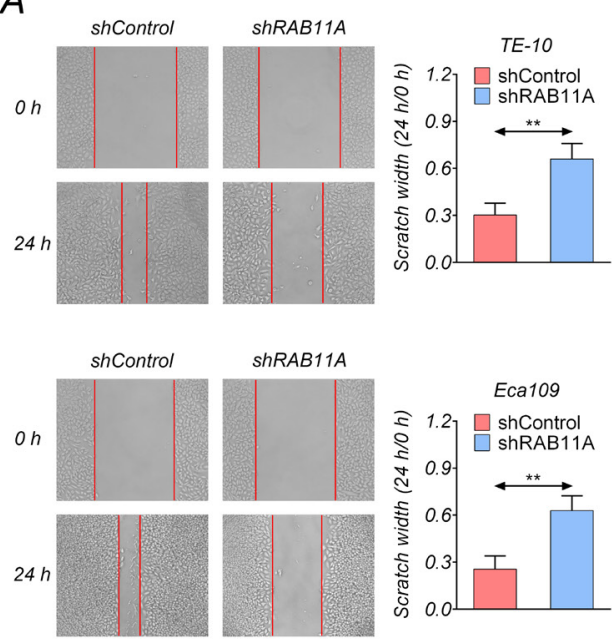

$B$

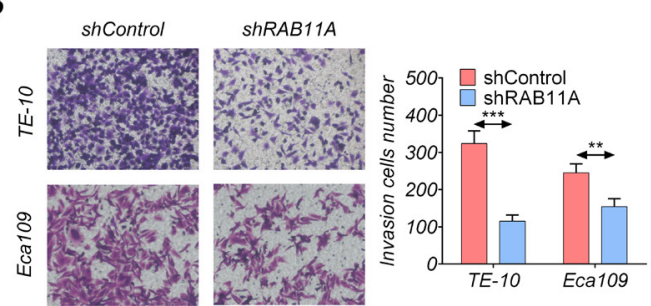

C

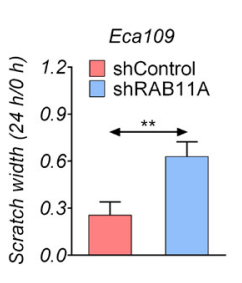

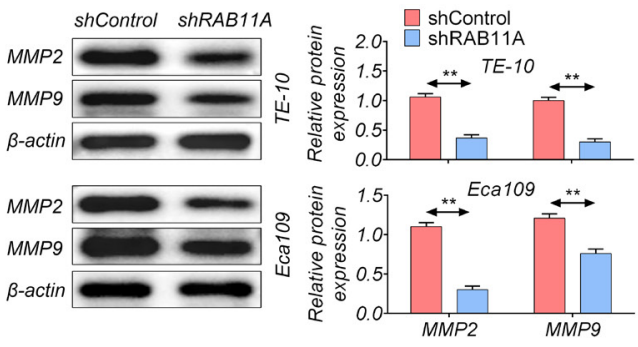

Figure 3. RAB11A promoted the migration and invasion of EC cells.

(A) Wound closure assays were performed to evaluate the migration of TE-10 or Eca109 cells. (B) Transwell assays were performed to measure the number of migrated TE-10 or Eca109 cells. (C) The expression of MMP2 and MMP9 in TE-10 or Eca109 cells was detected using immunoblot assays. All experiments were repeated three times, and results were presented as mean \pm S.D., **indicates $P<0.01$, ***indicates $P<0.001$. 

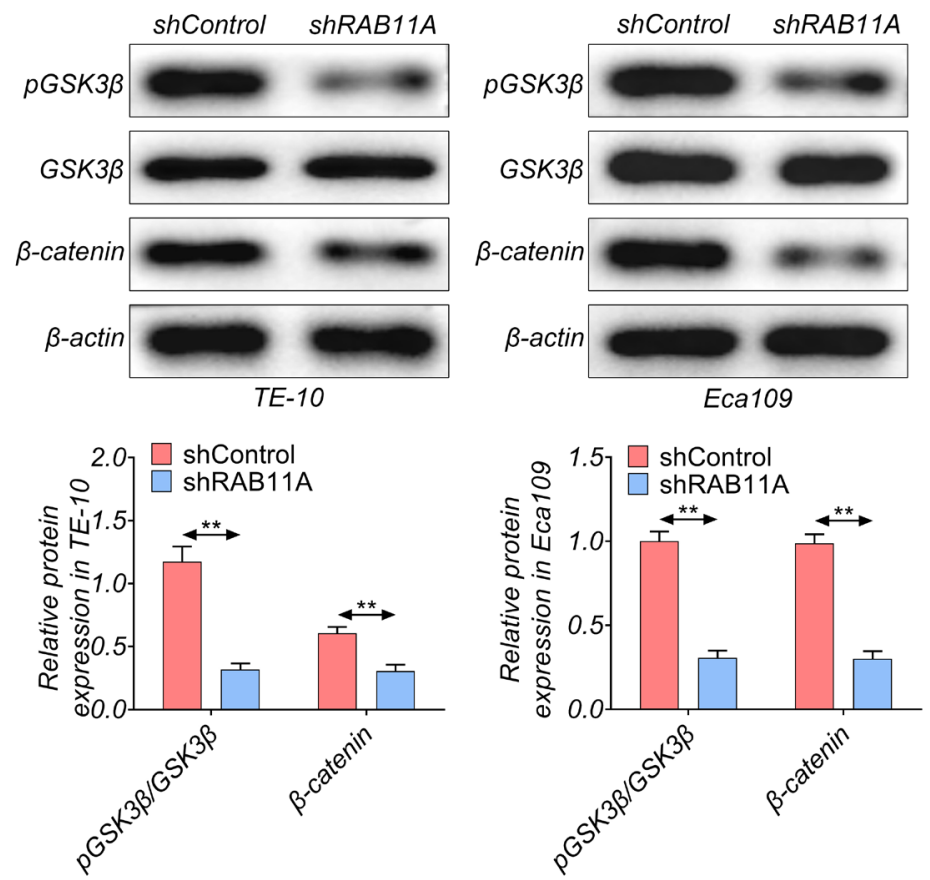

Figure 4. RAB11A depletion suppressed WNT pathway in EC cells.

The protein levels of GSK3 $\beta$, phosphorylated GSK3 $\beta$ (pGSK3 $\beta$ ), and $\beta$-catenin in TE-10 or Eca109 cells were detected using immunoblot assays. All experiments were repeated three times, and the results were presented as mean \pm S.D., ${ }^{* *}$ indicates $P<0.01$.

each year (Triantafyllou et al., 2020). Though surgical treatment is the most common treatment for early EC (Yamamoto et al., 2020), in patients with advanced EC high heterogeneity and metastasis are observed, targeted therapies have thus emerged as a new therapeutic direction (Xu et al., 2020). In order to improve the survival rate of patients with EC, it is still urgent to develop new EC therapeutic targets. Importantly, in the present study,
RAB11A was upregulated in human EC tissues and predicted poor prognosis. Moreover, the expression of RAB11A was associated with the clinical features, including pTNM stage and recurrence of patients with EC, suggesting that RAB11A was closely related to EC progression. Taken this data together, RAB11A might serve as a promising therapeutic target for EC treatment.
A

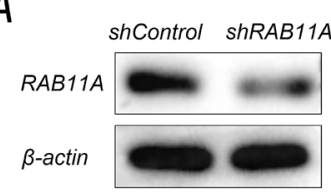

$B$

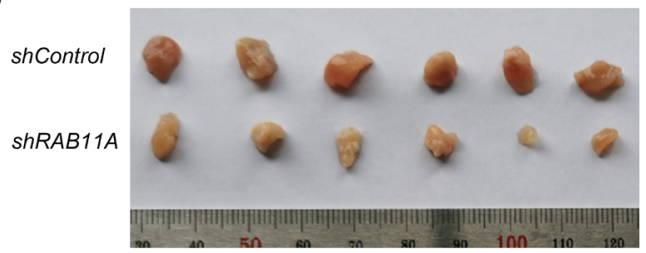

C

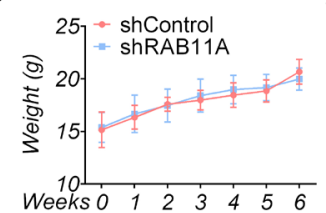

$D$

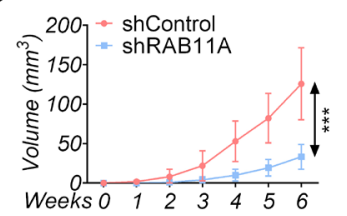

$E$
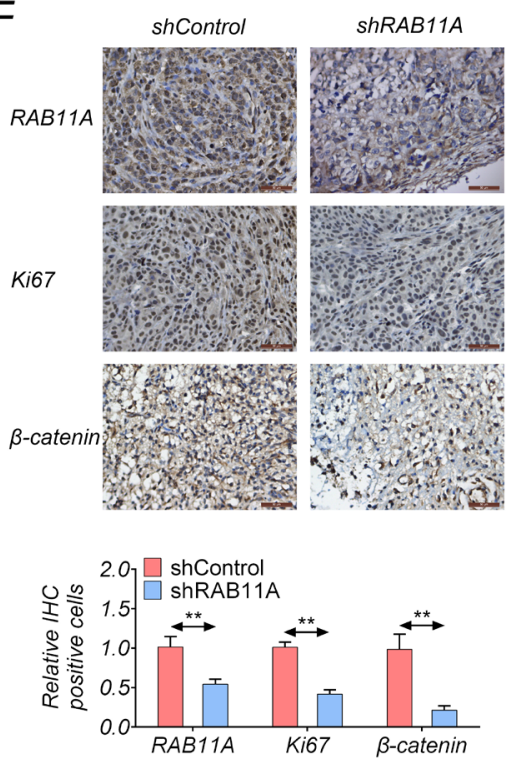

Figure 5. Knockdown of RAB11A suppressed EC tumor growth via WNT pathway in vivo.

(A) TE-10 cells infected with shRAB11A or shControl ( $n=6$ in each group) were implanted into nude mice. Tumor volume was monitored every week. After 6 weeks, tumors were isolated. The silencing efficiency of RAB11A shRNA was shown in panel (A), representative images of tumors were shown in panel (B). Mouse weight (C) and tumor growth curves (D) were calculated. (E) Immunohistochemical assays revealed the expression levels of RAB11A, Ki67 and $\beta$-catenin in tumor tissues of mice in shRAB11A or shControl group. Results were presented as mean \pm S.D., ${ }^{* *}$ indicates $P<0.01,{ }^{* * *}$ indicates $P<0.001$. 
Using MTT, colony formation, wound closure and Transwell assays, RAB11A was proved to promote the proliferation, colony formation, migration and invasion of EC cells. These in vitro experiments provided evidence showing that RAB11A contributed to the development of EC in a variety of regulatory pathways, which was also confirmed in the mice model of EC. Similarly, numerous studies indicated that RAB11A could act as an oncogene in multiple types of cancers, such as breast cancer, lung cancer, and pancreatic cancer (Palmieri et al., 2006; Khandelwal et al., 2013; Bai et al., 2017; Dong et al., 2017). A microRNA, miR-320a, suppressed the proliferation and migration of breast cancer cells via targeting RAB11A (Wang et al., 2015). Additionally, RAB11A was regulated by lnc-SNHG1 in invasive pituitary tumors (Wang et al., 2018). RAB11A also contributed to the proliferation and motility of NSCLC via regulating YAP (Dong et al., 2017). These investigations, together with our findings, indicated that RAB11A might act as a promising $\mathrm{EC}$ therapeutic target.

RAB11A is known as a member of Ras superfamily of small $G$ proteins which could mediate vesicle transport in cells (Kazemi-Noureini et al., 2004; Baetz \& Goldenring 2013). RAB11A promotes the recycling of a variety of receptors to the cell surface and thus affects multiple cellular processes, which suggests its regulatory effects on cancer progression (Kakar-Bhanot et al., 2019). RAB11A could also regulate E-cadherin transport and lumen formation, which also could potentially affect tumorigenesis (Khandelwal et al., 2008; Elias et al., 2015). Further analysis should clarify whether RAB11A promoted EC progression via similar mechanisms. It is widely known that WNT pathway is important for the development, invasion, and metastasis of multiple types of tumors, such as lung cancer, gastric cancer, and breast cancer $(\mathrm{Yu}$ et al., 2016). Notably, RAB11A promoted thyroid cancer growth via WNT/ $\beta$-Catenin pathway (Yu et al., 2016). Another study indicated that RAB11A sustained WNT pathway to promote the progression of pancreatic cancer. We, therefore, speculated that the involvement of RAB11A in EC progression was via WNT pathway (Bai et al., 2017; Wang et al., 2018). Importantly, multiple proteins affected the progression of EC via this pathway, suggesting that WNT pathway could serve as a promising therapeutic target for EC (Yu et al., 2016; Bai et al., 2017; Wang et al., 2018). Here, immunoblot assays showed that RAB11A regulated the WNT pathway in EC, which was also confirmed in mice model. This study thus revealed the regulatory mechanism underlying the role of RAB11A in EC progression.

In conclusion, this study showed the upregulation of RAB11A in human EC tissues and cells. The expression of RAB11A was correlated with poor prognosis and clinical characteristics of patients with EC. Furthermore, RAB11A promoted the proliferation, migration and invasion of EC cells via WNT pathway, which was also confirmed in vivo. Therefore, our findings indicated RAB11A could serve as a promising therapeutic target for EC.

\section{Acknowledgement}

Not applicable.

\section{Conflict of interest}

The authors declare that no conflict of interest is associated with this work.

\section{Funding}

Not applicable.

\section{Ethics approval and consent to participate}

Patients' consent was obtained before clinical material collection. All procedures performed in this study involving human participants were in accordance with the standards upheld by the Ethics Committee of The Second Hospital of Dalian Medical University and with those of the 1964 Helsinki Declaration and its later amendments for ethical research involving human subjects.

Animal assays performed in this study were approved by the Laboratory Animal Ethics Committee of Dalian Medical University.

\section{Availability of data and materials}

All data generated or analyzed during this study are included in this published article.

\section{Authors' contributions}

BW conceived and designed the experiments, DYZ analyzed and interpreted the results of the experiments, HWC performed the experiments.

\section{REFERENCES}

Esophageal Cancer Treatment (Adult) (PDQ(R)): Health Professional Version. (2002) PDQ Cancer Information Summaries. Bethesda (MD)

Baetz NW, Goldenring JR (2013) Rab11-family interacting proteins define spatially and temporally distinct regions within the dynamic Rab11a-dependent recycling system. Mol Biol Cell 24: 643-658. https:/ / doi.org/10.1091/mbc.E12-09-0659

Bai D, Sun H, Wang X, Lou H, Zhang J, Wang X, Jiang L (2017) MiR-150 inhibits cell growth in vitro and in vivo by restraining the RAB11A/WNT/beta-catenin pathway in thyroid cancer. Med Sci Monit 23: 4885-4894. https://doi.org/10.12659/msm.906997

Calhoun BC, Lapierre LA, Chew CS, Goldenring JR (1998) Rab11a redistributes to apical secretory canaliculus during stimulation of gastric parietal cells. Am J Physiol 275: C163-C170. https://doi. org/10.1152/ajpcell.1998.275.1.C163

Dobilienė D, Kèvalas R, Rudaitiš S, Masalskienè (2018) A prognostic value of early urinary biomarkers NGAL and IL-18 in critically ill children: a 10-year literature review. Signa Vitae 14: 14-19

Dong Q, Fu L, Zhao Y, Du Y, Li Q, Qiu X, Wang E (2017) Rab11a promotes proliferation and invasion through regulation of YAP in non-small cell lung cancer. Oncotarget 8: 27800-27811. https://doi. org/10.18632/ oncotarget.15359

Elias S, McGuire JR, Yu H, Humbert S (2015) Huntingtin is required for epithelial polarity through RAB11A-mediated apical trafficking of PAR3-aPKC. PLoS Biol 13: e1002142. https://doi.org/10.1371/ journal.pbio.1002142

Feng Q, Bonder EM, Engevik AC, Zhang L, Tyska MJ, Goldenring JR, Gao N (2017) Disruption of Rab8a and Rab11a causes formation of basolateral microvilli in neonatal enteropathy. J Cell Sci 130: 2491-2505. https://doi.org/10.1242/jcs.201897

Husebye H, Aune MH, Stenvik J, Samstad E, Skjeldal F, Halaas O, Nilsen NJ, Stenmark H, Latz E, Lien E, Mollnes TE, Bakke O, Espevik T (2010) The Rab11a GTPase controls Toll-like receptor 4-induced activation of interferon regulatory factor- 3 on phagosomes. Immunity 33: 583-596. https://doi.org/10.1016/j.immuni.2010.09.010

Jiang C, Liu Z, Hu R, Bo L, Minshall RD, Malik AB, Hu G (2017) Inactivation of Rab11a GTPase in macrophages facilitates phagocytosis of apoptotic neutrophils. J Immunol 198: 1660-1672. https:// doi.org/10.4049/jimmunol.1601495

Kakar-Bhanot R, Brahmbhatt K, Chauhan B, Katkam RR, Bashir T, Gawde H, Mayadeo N, Chaudhari UK, Sachdeva G (2019) Rab11a drives adhesion molecules to the surface of endometrial epithelial cells. Hum Reprod 34: 519-529. https://doi.org/10.1093/humrep/ dey365

Kazemi-Noureini S, Colonna-Romano S, Ziaee AA, Malboobi MA, Yazdanbod M, Setayeshgar P, Maresca B (2004) Differential gene expression between squamous cell carcinoma of esophageus and its normal epithelium; altered pattern of mal, akr1c2, and rab11a expression. World J Gastroenterol 10: 1716-1721. https://doi. org/10.3748/wig.v10.i12.1716

Khandelwal P, Prakasam HS, Clayton DR, Ruiz WG, Gallo LI, van Roekel D, Lukianov S, Peranen J, Goldenring JR, Apodaca G (2013) A Rab11a-Rab8a-Myo5B network promotes stretch-regulated 
exocytosis in bladder umbrella cells. Mol Biol Cell 24: 1007-1019. https://doi.org/10.1091/mbc.E12-08-0568

Khandelwal P, Ruiz WG, Balestreire-Hawryluk E, Weisz OA, Goldenring JR, Apodaca G (2008) Rab11a-dependent exocytosis of discoidal/fusiform vesicles in bladder umbrella cells. Proc Natl Acad Sci U S A 105: 15773-15778. https://doi.org/10.1073/pnas.0805636105

Knowles BC, Weis VG, Yu S, Roland JT, Williams JA, Alvarado GS, Lapierre LA, Shub MD, Gao N, Goldenring JR (2015) Rab11a regulates syntaxin 3 localization and microvillus assembly in enterocytes. J Cell Sci 128: 1617-1626. https://doi.org/10.1242/jcs.163303

Lutfi W, Martinez-Meehan D, Dhupar R, Christie N, Sarkaria I, Ekeke C, Baker N, Luketich JD, Okusanya OT (2020) Higher lymph node harvest in patients with a pathologic complete response after neoadjuvant therapy for esophageal cancer is associated with improved survival. J Surgical Oncol 121: 654-661

Nam SY, Jeon SW, Lee SJ, Kwon YH, Lee HS, Kim SK (2020) Clinical factors to predict the response to concurrent chemoradiotherapy and survival in esophageal cancer patients. Gut and Liver 14: 450458. https://doi.org/10.5009/gnl19165

Palmieri D, Bouadis A, Ronchetti R, Merino MJ, Steeg PS (2006) Rab11a differentially modulates epidermal growth factor-induced proliferation and motility in immortal breast cells. Breast Cancer Res Treat 100: 127-137. https://doi.org/10.1007/s10549-006-9244-6

Robert ME, Washington MK, Lee JR, Goldenring JR, Bronner MP, Goldblum JR, Greenson JK, Haber MM, Hart JA, Lamps LW, Lauwers GY, Lewin D, Lazenby AJ, Montgomery E, Crawford JM (2005) Rab11a immunohistochemical analysis does not distinguish indefinite, low-, and high-grade dysplasia in Barrett esophagus. Am J Clin Pathol 124: 519-527. https://doi. org/10.1309/7X4GAXT15H50B48P

Shrestha R, Tripathi R, Goyal P, Agarwal C, Gupta M, Mehta A, Doval DC (2020) Long-term survival in an esophageal cancer patient with multiple recurrences. J Gastrointest Cancer 51: 695-697. https:// doi.org/10.1007/s12029-020-00366-3

Su HA, Hsiao SW, Hsu YC, Wang LY, Yen HH (2020) Superiority of NBI endoscopy to PET/CT scan in detecting esophageal cancer among head and neck cancer patients: a retrospective cohort analysis. BMC Cancer 20: 69. https://doi.org/10.1186/s12885-020-6558-4

Triantafyllou T, Olson MT, Theodorou D, Schizas D, Singhal S (2020) Enhanced recovery pathways $v s$ standard care pathways in esophageal cancer surgery: systematic review and meta-analysis. Esophagus $1-13$

Wang B, Yang Z, Wang H, Cao Z, Zhao Y, Gong C, Ma L, Wang $\mathrm{X}, \mathrm{Hu} \mathrm{X}$, Chen S (2015) MicroRNA-320a inhibits proliferation and invasion of breast cancer cells by targeting RAB11A. Am J Cancer Res 5: 2719-2729

Wang $\mathrm{H}$, Wang G, Gao Y, Zhao C, Li X, Zhang F, Jiang C, Wu B (2018) Lnc-SNHG1 activates the TGFBR2/SMAD3 and RAB11A/ Wnt/beta-catenin pathway by sponging mir-302/372/373/520 in invasive pituitary tumors. Cell Physiol Biochem 48: 1291-1303. https:// doi.org/10.1159/000492089

Wu J, Liu L, Wu F, Qiu L, Luo M, Ke Q, Deng X, Luo Z (2020) Clinical and prognostic implications of P21 (WAF1/CIP1) expression in patients with esophageal cancer: a systematic review and meta-analysis. Dis Markers 2020: 6520259. https://doi. org/10.1155/2020/6520259

Xu J, Peng Y, Yang M, Guo N, Liu H, Gao H, Niu F, Wang R, Wang C, Yu K (2020) Increased levels of myeloid-derived suppressor cells in esophageal cancer patients is associated with the complication of sepsis. Biomed Pharmacother 125: 109864. https://doi.org/10.1016/j. biopha.2020.109864

Yamamoto K, Makino T, Sato E, Noma T, Urakawa S, Takeoka T, Yamashita K, Saito T, Tanaka K, Takahashi T (2020) Tumor-infiltrating M2 macrophage in pretreatment biopsy sample predicts response to chemotherapy and survival in esophageal cancer. Cancer Science 111: 1103.

Yu L, Li X, Li H, Chen H, Liu H (2016) Rab11a sustains GSK3beta/Wnt/beta-catenin signaling to enhance cancer progression in pancreatic cancer. Tumour Biol 37: 13821-13829. https://doi. org/10.1007/s13277-016-5172-1 